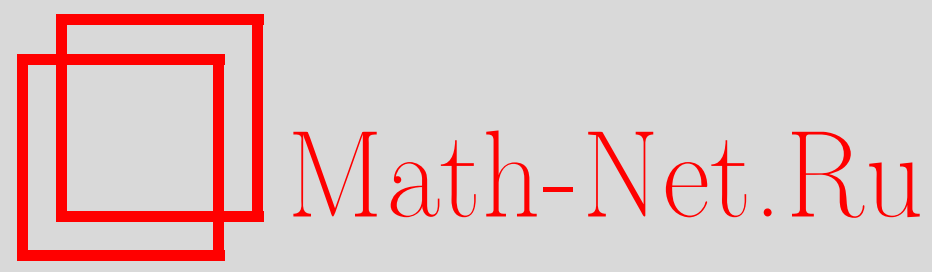

А. П. Буслаев, В. А. Кондратьев, А. И. Назаров, Об одном семействе экстремальных задач и связанных с ним свойствах одного интеграла, Матем. заметки, 1998, том 64, выпуск 6, 830-838

DOI: https://doi.org/10.4213/mzm1462

Использование Общероссийского математического портала Math-Net.Ru подразумевает, что вы прочитали и согласны с пользовательским соглашением http://www . mathnet.ru/rus/agreement

Параметры загрузки:

IP : 3.89 .185 .249

26 апреля 2023 г., 10:23:28

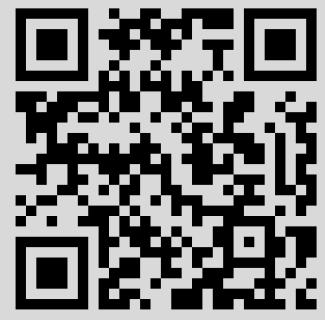




\section{ОБ ОДНОМ СЕМЕЙСТВЕ ЭКСТРЕМАЛЬНЫХ ЗАДАЧ И СВЯЗАННЫХ С НИМ СВОЙСТВАХ ОДНОГО ИНТЕГРАЛА}

\section{А. П. Буслаев, В. А. Кондратьев, А.И. Назаров}

Исследуется экстремальная задача

$$
\int_{0}^{1}\left(y^{\prime \prime}(t)\right)^{p} d t / \int_{0}^{1}\left(y^{\prime}(t)\right)^{q} d t \rightarrow \min
$$

по всем $y, y(0)=y(1)=0, y^{\prime}(0)=y^{\prime}(1)=0$, которая приводит к интегралу

$$
\int_{\mathbb{R}}\left(\max \left(0,1+\mu x-|x|^{q}\right)\right)^{1 / p^{\prime}} d x
$$

и дает точные оценки для собственных значений дифференциальных операторов в обобщенной задаче Лагранжа об устойчивости колонны.

Библиографой: 10 названий.

1. Постановка задачи. Пусть $1 \leqslant p, q \leqslant \infty$.

Рассмотрим следующую экстремальную задачу:

$$
\frac{\left\|y^{\prime \prime}\right\|_{L_{p}[0,1]}}{\left\|y^{\prime}\right\|_{L_{q}[0,1]}} \rightarrow \min
$$

при условии

$$
y(0)=y(1)=0, \quad y^{\prime}(0)=y^{\prime}(1)=0 .
$$

Ее решение дает точные оценки для собственных значений дифференциальных операторов в задаче Лагранжа об устойчивости колонны [1]-[5].

Стандартные рассуждения позволяют установить, что решение рассматриваемой задачи $(1),(2)$ существует, причем экстремаль можно искать среди функций $y$ с одной переменой знака производной $y^{\prime}$.

Замена $y^{\prime}(t)=x(t)$ приводит к задаче

$$
\begin{gathered}
\frac{\left\|x^{\prime}\right\|_{L_{p}[0,1]}}{\|x\|_{L_{q}[0,1]}} \rightarrow \min , \\
\int_{0}^{1} x(t) d t=0, \quad x(0)=x(1)=0 .
\end{gathered}
$$

Работа выполнена при поддержке Российского фонда фундаментальных исследований, гранты № 96-01-00325 (первый автор), № 96-15-96177 (второй автор), № 96-01-00087 (третий автор).

(C) А. П. БуСлАев, В. А. КОНдрАтьев, А. И. НАЗАрОв 1998 
Задача (3), (4) возникает также в математической статистике при исследовании условий локальной асимптотической оптимальности непараметрических статистических критериев [6].

В силу однородности функционала (3) можно считать, что $x$ нормирована в $L_{q}[0,1]$ :

$$
\|x\|_{L_{q}[0,1]}=1
$$

Пока будем считать, что $1<p, q<\infty$.

Необходимые условия экстремума в (3), (4) (см. [7]) приводят к нелинейной краевой задаче

$$
\left(\left|x^{\prime}(t)\right|^{p-1} \operatorname{sgn} x^{\prime}(t)\right)^{\prime}+\lambda|x(t)|^{q-1} \operatorname{sgn} x(t)=c_{1}, \quad t \in[0,1], \quad x(0)=x(1)=0
$$

при условии

$$
\int_{0}^{1} x(t) d t=0
$$

причем значение функционала (3) на решении задачи $(6),(7)$ равно $\lambda^{1 / p}$.

Интегрируя (6), получаем

$$
\frac{\left|x^{\prime}\right|^{p}}{p^{\prime}}+\lambda \frac{|x|^{q}}{q}=c_{1} x+c_{2}
$$

где $p^{\prime}=p /(p-1)$.

Интегрируя (8) по отрезку $[0,1]$, из условий $(5)$ и (7) получаем $c_{2}=\lambda\left(1 / p^{\prime}+1 / q\right)$.

$\mathrm{C}$ помощью подходящего преобразования вида $a x(b t)$ и переобозначения постоянной в (8), приходим к уравнению

$$
\left|x^{\prime}\right|^{p}=1+\mu x-|x|^{q}
$$

при условиях $x(0)=x(T)=0$,

$$
\int_{0}^{T} x(t) d t=0
$$

Уравнение (9) задает в фазовой плоскости $\left(x, x^{\prime}\right)$ овал, причем, поскольку на $(0, T) x$ меняет знак один раз, $T$ есть главньй период движения по этому овалу.

Очевидно, что при $\mu=0$ овал (9) симметричен относительно обеих координатных осей в фазовой плоскости, и условие (10) выполнено автоматически. При этом решение задачи $(6),(7)$ центрально симметрично относительно точки $(1 / 2,0)$. Если же $\mu \neq 0$, то решение такой симметрией не обладает.

Заметим, что в случае $p=2, q=2 n+1, n \in \mathbb{N}$, решение уравнения (9) при $\mu=0$ (так называемый обобщенный синус) исследовалось А. М. Ляпуновым [8].

Поскольку овал (9) симметричен относительно оси $x^{\prime}$, условие (10) можно заменить на условие

$$
\int_{t_{1}}^{t_{2}} x(t) d t=0
$$

где $t_{1}$ и $t_{2}$ - соответственно точки минимума и максимума решения $(9)$ на $[0, T]$ (заметим, что $\left.\left|t_{2}-t_{1}\right|=T / 2\right)$. 
Подставляя в $(11) d t=d x /\left(1+\mu x-|x|^{q}\right)^{1 / p}($ см. (9)), получим уравнение

$$
F(p, q, \mu) \equiv \int_{x_{1}}^{x_{2}} \frac{x d x}{\left(1+\mu x-|x|^{q}\right)^{1 / p}}=0
$$

где $x_{1}, x_{2}$ - вещественные нули функции $1+\mu x-|x|^{q}, x_{1}=x_{1}(\mu)<x_{2}=x_{2}(\mu)$. Легко видеть, что $x_{2}(-\mu)=-x_{1}(\mu)$ и $F-$ нечетная по $\mu$ функция. Очевидно также, что при $\mu \geqslant 0$ имеет место $-1 \leqslant x_{1}(\mu)<0<x_{2}(\mu)$.

Рассмотрим интеграл

$$
J(p, q, \mu)=\int_{x_{1}}^{x_{2}}\left(1+\mu x-|x|^{q}\right)^{1 / p^{\prime}} d x
$$

как функцию переменной $\mu$ с параметрами $p, q$.

Очевидно, что $J_{\mu}^{\prime}=F(p, q, \mu) / p^{\prime}$. Поэтому точки минимума $J$ удовлетворяют уравнению (12).

Далее, если $x(t)$ - решение уравнения $(9)$, то

$$
J(p, q, \mu)=\int_{0}^{T}\left|x^{\prime}\right|^{p} d t .
$$

Возвращаясь вновь к отрезку $[0,1]$ и нормируя решение, получаем

$$
J(p, q, \mu)=\frac{1}{2} \Theta^{-\Theta} q^{-1 / q} p^{\prime-1 / p^{\prime}} \lambda^{1 / p}
$$

где $\Theta=1 / q+1 / p^{\prime}$. Поэтому минимум в задаче $(3),(4)$ равен

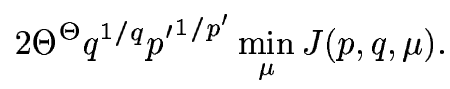

2. Исследование уравнения (12). Как уже отмечалось, $F(p, q, 0)=0$ при всех $p, q$. Поэтому вопрос о ненулевых решениях уравнения (12) приводит к задаче о бифуркации нулевого решения [9].

Будем решать уравнение бифуркации $F_{\mu}^{\prime}(p, q, 0)=0$.

Теорема 1. Для любы $x \mu \in \mathbb{R}, 1<q, p<\infty$

$$
F_{\mu}^{\prime}(p, q, \mu)=-\left(\frac{q}{p}-3\right) I_{1}-\mu(q-1) I_{2},
$$

əəe

$$
\begin{aligned}
& I_{1}=I_{1}(p, q, \mu)=\int_{x_{1}}^{x_{2}} \frac{x^{2} d x}{\left(1+\mu x-|x|^{q}\right)^{1 / p}(q+(q-1) \mu x)} \\
& I_{2}=I_{2}(p, q, \mu)=\int_{x_{1}}^{x_{2}} \frac{x^{3} d x}{\left(1+\mu x-|x|^{q}\right)^{1 / p}(q+(q-1) \mu x)^{2}}
\end{aligned}
$$


ДокАЗАТЕЛЬСтво. Имеем

$$
f_{\varepsilon}(\mu) \equiv \int_{x_{1}+\varepsilon}^{x_{2}-\varepsilon} \frac{x d x}{\left(1+\mu x-|x|^{q}\right)^{1 / p}} \rightarrow F(p, q, \mu), \quad \varepsilon \rightarrow 0
$$

причем сходимость равномерная на любом конечном промежутке. Далее,

$$
\begin{aligned}
\frac{d f_{\varepsilon}}{d \mu}= & \left.\frac{d x_{2}}{d \mu} \frac{x}{\left(1+\mu x-|x|^{q}\right)^{1 / p}}\right|^{x_{2}-\varepsilon}-\left.\frac{d x_{1}}{d \mu} \frac{x}{\left(1+\mu x-|x|^{q}\right)^{1 / p}}\right|^{x_{1}+\varepsilon} \\
& -\frac{1}{p} \int_{x_{1}+\varepsilon}^{x_{2}-\varepsilon} \frac{x^{2} d x}{\left(1+\mu x-|x|^{q}\right)^{1 / p+1}}
\end{aligned}
$$

Однако, из $1+\mu x_{k}-\left|x_{k}\right|^{q}=0, k=1,2$, имеем

$$
\frac{d x_{k}}{d \mu}=\frac{x_{k}}{q\left|x_{k}\right|^{q-2} x_{k}-\mu}=\frac{x_{k}^{2}}{q+(q-1) \mu x_{k}} .
$$

Таким образом,

$$
\begin{aligned}
\frac{d f_{\varepsilon}}{d \mu}= & \left.\frac{x^{3}}{\left(1+\mu x-|x|^{q}\right)^{1 / p}(q+(q-1) \mu x)}\right|_{x_{1}+\varepsilon} ^{x_{2}-\varepsilon} \\
& +O\left(\varepsilon^{1 / p^{\prime}}\right)-\frac{1}{p} \int_{x_{1}+\varepsilon}^{x_{2}-\varepsilon} \frac{x^{2} d x}{\left(1+\mu x-|x|^{q}\right)^{1 / p+1}} \\
= & \int_{x_{1}+\varepsilon}^{x_{2}-\varepsilon}\left(\frac{d}{d x}\left(\frac{x^{3}}{\left(1+\mu x-|x|^{q}\right)^{1 / p}(q+(q-1) \mu x)}\right)\right. \\
& \left.-\frac{1}{p} \frac{x^{2}}{\left(1+\mu x-|x|^{q}\right)^{1 / p+1}}\right) d x+O\left(\varepsilon^{1 / p^{\prime}}\right) \\
= & \int_{x_{1}+\varepsilon}^{x_{2}-\varepsilon}\left(\frac{-x^{2}(q / p-3)}{\left(1+\mu x-|x|^{q}\right)^{1 / p}(q+(q-1) \mu x)}\right. \\
& \left.-\frac{(q-1) \mu x^{3}}{\left(1+\mu x-|x|^{q}\right)^{1 / p}(q+(q-1) \mu x)^{2}}\right) d x+O\left(\varepsilon^{1 / p^{\prime}}\right) .
\end{aligned}
$$

При $\varepsilon \rightarrow 0 d f_{\varepsilon} / d \mu \rightarrow-(q / p-3) I_{1}-(q-1) \mu I_{2}$, где сходимость также равномерна на любом конечном промежутке, что и доказьвает теорему.

Из теоремы 1 видно, что при фиксированном $p$ решением уравнения бифуркации будет $q=3 p$. Поскольку $F_{\mu q}^{\prime \prime}(p, 3 p, 0)=-I_{1} / p<0$, в этой точке вьполнено достаточное условие бифуркации [9] и от нулевого решения уравнения (12) ответвляется ненулевая ветка. В силу нечетности $F$, если $\mu$ - решение (12), то и - $\mu$ - также решение (12).

Разложение функции $F$ в окрестности точки бифуркации имеет вид

$$
F(p, q, \mu)=\frac{1}{6} F_{\mu \mu \mu}^{\prime \prime \prime}(p, 3 p, 0) \mu^{3}-I_{1} \mu(q-3 p)+\cdots
$$

Вычисление, аналогичное теореме 1 , дает

$$
F_{\mu \mu \mu}^{\prime \prime \prime}(p, 3 p, 0)=-(q-1) I_{2 \mu}^{\prime}(p, 3 p, 0)=\frac{2(3 p-2)(3 p-1)}{27 p^{3}} \int_{-1}^{1} \frac{x^{4} d x}{\left(1-|x|^{3 p}\right)^{1 / p}}>0 .
$$

Поэтому ненулевое решение локально существует только при $q>3 p$, и модуль его растет с ростом $q$. 
TeOpema 2. Пусть $1<p, q<\infty$. Тогда

а) при $q>3 p$ уравнение (12) имеет положительное решение;

б) существует $\varepsilon>0$ такое, что при $q \leqslant 2 p+\varepsilon$ уравнение (12) не имеет ненулевых решений.

ДокАЗАТЕЛЬСТво. а) Поскольку в формуле (14) $I_{1}>0$, то при $q>3 p F_{\mu}^{\prime}<0$, поэтому $F(p, q, \mu)<0$ для малых положительных $\mu$.

Далее, при $\mu \rightarrow \infty$ справедливо $x_{1}(\mu) \rightarrow-0, x_{2}(\mu) \rightarrow \infty$. Поэтому

$$
\begin{gathered}
\int_{x_{1}}^{0} \frac{x d x}{\left(1+\mu x-|x|^{q}\right)^{1 / p}} \sim \int_{-1 / \mu}^{0} \frac{x d x}{(1+\mu x)^{1 / p}}=-\mu^{-2} \int_{0}^{1} \frac{t d t}{(1-t)^{1 / p}} \\
\int_{0}^{x_{2}} \frac{x d x}{\left(1+\mu x-|x|^{q}\right)^{1 / p}} \sim \int_{0}^{\mu^{1 /(q-1)}} \frac{x d x}{\left(\mu x-|x|^{q}\right)^{1 / p}} \\
=\mu^{2 /(q-1)-q /(p(q-1))} \int_{0}^{1} \frac{t d t}{\left(t-|t|^{q}\right)^{1 / p}}
\end{gathered}
$$

Поскольку при рассматриваемых значениях $p, q$ показатель степени при $\mu$ в (17) больше, чем в (16), то при $\mu \gg 1 F(p, q, \mu)>0$, что доказьвает утверждение а).

ЗАмЕчАнИЕ 1 . Из доказательства утверждения а) видно, что при $q \leqslant 3 p F(\mu)$ не обращается в 0 как в окрестности нуля, так и в окрестности бесконечности.

б) Перепишем формулу (14) в виде интеграла

$$
\begin{aligned}
G(p, q, \mu):=F_{\mu}^{\prime}(p, q, \mu) & =\frac{1}{p} \int_{x_{1}}^{x_{2}} \frac{x^{2}(q(3-q / p)+(q-1) \mu x(2-q / p)) d x}{\left(1+\mu x-x^{q}\right)^{1 / p}(q+(q-1) \mu x)^{2}} \\
& =\frac{r}{p q} \int_{x_{1}}^{x_{2}} \frac{x^{2}\left(1+\mu x /\left(q^{\prime} r^{\prime}\right)\right) d x}{\left(1+\mu x-|x|^{q}\right)^{1 / p}\left(1+\mu x / q^{\prime}\right)^{2}}
\end{aligned}
$$

где $r=3-p / q$. При $q \leqslant 2 p$ подынтегральная функция неотрищательна на $\left[x_{1}, x_{2}\right]$. Следовательно, $F(p, q, \mu)$ отделена от нуля на любом промежутке $[m, M], m>0$. По непрерьвности $F$ не обрашается в 0 при $q \leqslant 2 p+\varepsilon$, что и завершает доказательство.

ЗАмЕчАниЕ 2. В работе [10] получены более слабые достаточные условия сушествования и отсутствия ненулевого решения уравнения (12).

3. Поведение функций $J(p, q, \mu), F(p, q, \mu), G(p, q, \mu)$. Из теоремы 2 следует, что при фиксированных $p, q, 1<q, p<\infty, q>3 p$, точка $\mu=0$ дает функционалу $J$ локальньй максимум; а поскольку $J$ возрастает при больших $\mu$, минимум достигается на ненулевом решении уравнения (12). При $q \leqslant 3 p \quad \mu=0$ является по крайней мере точкой локального минимума $J$, а при $q \leqslant 2 p$ - даже глобального.

При $\mu \rightarrow \infty$ из (16) и (17) следует, что

$$
F(\mu)=\mu^{(r-1) /(q-1)}\left(\int_{0}^{1} \frac{t d t}{\left(t-|t|^{q}\right)^{1 / p}}+\cdots\right) .
$$

Таким образом, при $r \in[0,1) F(\mu)$ возрастает в окрестности нуля и убьвает в окрестности бесконечности. Отсюда следует, что при $r \in[0,1) F_{\mu}^{\prime}(\mu)$ имеет хотя бы один положительньй корень. При $r \geqslant 1$, как показано в теореме $2, F_{\mu}^{\prime}(\mu)$ корней не имеет. 
ГИПотеЗА 1. Нуль является точкой глобального минимума функиионала $J$ при всех $q \leqslant 3 p$.

ГИПотЕЗА 2. Уравнение (12) имеет положительное решение только при $q>3 p$, причем это решение единственно и является возрастающей функиией $q$ при фиксированном р.

4. Точные значения $J(p, q, \mu)$. Известно, что

$$
\begin{aligned}
J(p, q, 0) & =\int_{-1}^{1}\left(1-|x|^{q}\right)^{1 / p^{\prime}} d x=\frac{2}{q} \mathrm{~B}\left(\frac{1}{q}, 1+\frac{1}{p^{\prime}}\right) \\
& =\frac{2}{q} \frac{\Gamma(1 / q) \Gamma\left(1+1 / p^{\prime}\right)}{\Gamma\left(1 / q+1 / p^{\prime}+1\right)}=\frac{2}{p^{\prime} q} \frac{\Gamma(1 / q) \Gamma\left(1 / p^{\prime}\right)}{\Gamma\left(1+1 / p^{\prime}+1 / q\right)},
\end{aligned}
$$

где $\Gamma$ - гамма-функция. Функция $J(p, q, 0)$ симметрична относительно $p^{\prime}, q$ и монотонна по каждому аргументу при $p \geqslant 1, q \geqslant 1$.

Рассмотрим несколько случаев.

1) Случай $q=1$. Имеет место

$$
J(p, 1, \mu)= \begin{cases}(2 p)^{\prime} \cdot \frac{1}{1-\mu^{2}}, & \text { если } 0 \leqslant \mu<1, \\ \infty, & \text { если } \mu \geqslant 1 .\end{cases}
$$

Действительно,

$$
\begin{aligned}
J(p, 1, \mu) & =\int_{1+\mu x-|x| \geqslant 0}(1+\mu x-|x|)^{1 / p^{\prime}} d x \\
& =\int_{x_{1}}^{0}(1+\mu x+x)^{1 / p^{\prime}} d x+\int_{0}^{x_{2}}(1+\mu x-x)^{1 / p^{\prime}} d x \\
& =\int_{-1 /(\mu+1)}^{0}(1+(\mu+1) x)^{1 / p^{\prime}} d x+\int_{0}^{1 /(1-\mu)}(1+(\mu-1) x)^{1 / p^{\prime}} d x \\
& =\frac{1}{1+1 / p^{\prime}}\left(\frac{1}{\mu+1}+\frac{1}{1-\mu}\right)=(2 p)^{\prime} \cdot \frac{1}{1-\mu^{2}}, \quad 0<\mu<1 .
\end{aligned}
$$

Вторая часть утверждения очевидна.

2) Случай $q=2$. Имеет место

$$
J(p, 2, \mu)=\mathrm{B}\left(\frac{1}{2}, 1+\frac{1}{p^{\prime}}\right)\left(\frac{\mu^{2}}{4}+1\right)^{\Theta},
$$

где $\Theta=1 / 2+1 / p^{\prime}$.

Действительно,

$$
\begin{aligned}
J(p, 2, \mu) & =\int_{x_{1}(\mu)}^{x_{2}(\mu)}\left(\left(x_{2}(\mu)-x\right)\left(x-x_{2}(\mu)\right)\right)^{1 / p^{\prime}} d x \\
& =\left(\frac{x_{2}(\mu)-x_{1}(\mu)}{2}\right)^{1+2 / p^{\prime}} \int_{-1}^{1}\left(1-t^{2}\right)^{1 / p^{\prime}} d t=\mathrm{B}\left(\frac{1}{2}, 1+\frac{1}{p^{\prime}}\right)\left(\frac{\mu^{2}}{4}+1\right)^{\Theta},
\end{aligned}
$$


поскольку

$$
x_{1,2}(\mu)=\frac{\mu}{2} \pm \sqrt{\frac{\mu^{2}}{4}+1}, \quad x_{2}(\mu)-x_{1}(\mu)=2 \sqrt{\frac{\mu^{2}}{4}+1} .
$$

3) Случай $q=\infty$. При $0<\mu \leqslant 1$ имеем

$$
J(p, \infty, \mu)=\lim _{q \rightarrow \infty} J(p, q, \mu)=\int_{-1}^{1}(1+\mu x)^{1 / p^{\prime}} d x=\frac{(1+\mu)^{1+1 / p^{\prime}}}{\left(1+1 / p^{\prime}\right) \mu}-\frac{(1-\mu)^{1+1 / p^{\prime}}}{\left(1+1 / p^{\prime}\right) \mu} .
$$

Отсюда

$$
F(\mu)=\frac{d J}{d \mu}=\frac{1}{\mu^{2}\left(1+1 / p^{\prime}\right)}\left(\left(\frac{\mu}{p^{\prime}}-1\right)(1+\mu)^{1 / p^{\prime}}+\left(\frac{\mu}{p^{\prime}}+1\right)(1-\mu)^{1 / p^{\prime}}\right) .
$$

Очевидно, что при $p=1$ и $p=\infty \quad F \equiv 0$. Рассмотрим случай $1<p<\infty$.

Заметим, что при разложении выражения

$$
\left(\frac{\mu}{p^{\prime}}-1\right)(1+\mu)^{1 / p^{\prime}}+\left(\frac{\mu}{p^{\prime}}+1\right)(1-\mu)^{1 / p^{\prime}}
$$

в степенной ряд по $\mu$ коэффициенты при $1, \mu, \mu^{2}$ обращаются в 0 . Поэтому $F(0)=0$.

Далее, при $0<\mu \leqslant 1$

$$
\left(\mu^{2} F(\mu)\right)^{\prime}=\frac{\mu}{p^{\prime}}\left((1+\mu)^{1 / p^{\prime}-1}-(1-\mu)^{1 / p^{\prime}-1}\right)<0
$$

и, следовательно, $F(\mu)<0$. Если $\mu>1$, то

$$
J(p, \infty, \mu)=\int_{-1 / \mu}^{1}(1+\mu x)^{1 / p^{\prime}} d x=\frac{(1+\mu)^{1+1 / p^{\prime}}}{\left(1+1 / p^{\prime}\right) \mu} .
$$

Далее,

$$
\frac{d J}{d \mu}=\frac{(1+\mu)^{1 / p^{\prime}}}{\mu^{2}\left(1+1 / p^{\prime}\right)}\left(\frac{\mu}{p^{\prime}}-1\right)
$$

очевидно, обращается в 0 при $\mu=p^{\prime}$. При этом прямьм вычислением можно показать, что минимум в соответствующей задаче $(3),(4)$, равньй $2\left(1+p^{\prime}\right)^{1 / p^{\prime}}$, достигается на ломаной экстремали

$$
x(t)=\frac{1}{p^{\prime}} \cdot \begin{cases}\left|2\left(1+p^{\prime}\right)^{1 / p^{\prime}} t-1\right|^{p^{\prime}}-1, & \text { если } 0 \leqslant t \leqslant t_{0}, \\ \left|2\left(1+p^{\prime}\right)^{1 / p^{\prime}}(t-1)-1\right|^{p^{\prime}}-1, & \text { если } t_{0} \leqslant t \leqslant 1,\end{cases}
$$

где $t_{0}=\left(1+\left(1+p^{\prime}\right)^{-1 / p^{\prime}}\right) / 2$.

4) Случай $p=1, q<\infty$. Из формулы (13) имеем

$$
J(1, q, \mu):=\lim _{p \rightarrow 1} J(p, q, \mu)=x_{2}(\mu)-x_{1}(\mu) .
$$


Из формул (15) вытекает, что $x_{1}^{\prime}(0)=x_{2}^{\prime}(0)=1 / q$, а дифференцируя (15) три раза, получаем

$$
\begin{gathered}
x_{1}^{\prime \prime}(0)=-x_{2}^{\prime \prime}(0)=\frac{q-3}{q^{2}}, \quad x_{1}^{\prime \prime \prime}(0)=x_{2}^{\prime \prime \prime}(0)=\frac{2(q-4)(q-2)}{q^{3}} \\
x_{1}^{(4)}(0)=-x_{2}^{(4)}(0)=\frac{(q-5)(2 q-5)(3 q-5)}{q^{4}}
\end{gathered}
$$

Поэтому при мальх $\mu$

$$
x_{2}(\mu)-x_{1}(\mu)=2-\mu^{2} \frac{q-3}{q^{2}}+\mu^{4} \frac{(5-q)(2 q-5)(3 q-5)}{12 q^{4}}+\cdots .
$$

Таким образом, как и при $p>1$, нуль является для $J$ точкой локального максимума при $q>3 p$ и локального минимума при $q \leqslant 3 p$.

5) Случай $p=\infty, q<\infty$. Имеем

$$
J(\infty, q, \mu)=\int_{x_{1}(\mu)}^{x_{2}(\mu)}\left(1+\mu x-|x|^{q}\right) d x=\left.\left(x+\mu \frac{x^{2}}{2}-\frac{|x|^{q} x}{q+1}\right)\right|_{x_{1}(\mu)} ^{x_{2}(\mu)} .
$$

Выражая последнее слагаемое из уравнения для $x_{1}$ и $x_{2}$, получаем

$$
J(\infty, q, \mu)=\frac{q}{q+1}\left(x_{2}(\mu)-x_{1}(\mu)\right)+\frac{(q-1) \mu}{2(q+1)}\left(x_{2}^{2}(\mu)-x_{1}^{2}(\mu)\right) .
$$

Дифференцируя под знаком интеграла, получаем

$$
\frac{d J}{d \mu}=\int_{x_{1}(\mu)}^{x_{2}(\mu)} x d x=\frac{\left(x_{2}(\mu)\right)^{2}-\left(x_{1}(\mu)\right)^{2}}{2} .
$$

Из (15) видно, что $x_{1}$ и $x_{2}$ монотонно возрастают по $\mu$. Поскольку $x_{1}(0)=-1$, $x_{2}(0)=1$, при $\mu>0$ выполнено $d J / d \mu>0$.

5. Более общая постановка и геометрический смысл функционала $J$. Рассмотрим следующее уравнение $(1<p, q<\infty)$ :

$$
|x|^{q}+|y|^{p}=a x+b y+1
$$

которое определяет на плоскости $(x, y)$ выпуклую замкнутую кривую, являющуюся проекцией на эту плоскость линии пересечения поверхностей

$$
z_{1}=|x|^{q}+|y|^{p}, \quad z_{2}=a x+b y+1 .
$$

Ясно, что для исследования зависимости от $a$ и $b$ площадей $S(p, q, a, b)$ (при этом полагаем $\left.S(p, q, a, 0) \equiv 2 J\left(p^{\prime}, q, a\right)\right)$, ограниченных этими кривьми, достаточно считать $a \geqslant 0, b \geqslant 0$.

В частности, при $p=q=2$ получаем двупараметрическое семейство окружностей $x^{2}+y^{2}=a x+b y+1$. Таким образом,

$$
S(2,2, a, b)=\pi\left(1+\frac{a^{2}}{4}+\frac{b^{2}}{4}\right) .
$$

Аналогично п. 3 зададим вопрос в более общей постановке: исследовать монотонность $S(p, q, a, b)$ в зависимости от аргументов $a, b$ при фиксированных $p, q$. 


\section{СПИСОК ЦИТИРОВАННОЙ ЛИТЕРАТУРЫ}

[1] Keller J. B. The shape of the strongest column // Arch. Rational Mech. Anal. 1960. V. 5. P. 275-285.

[2] Егоров Ю. В., Кондратьев В.А. Об оценках первого собственного значения задачи Штурма-Лиувилля // УМН. 1984. Т. 39. № 2. С. 151-152.

[3] Егоров Ю. В., Кондратьев В. А. Об оценке первого собственного значения оператора Штурма-Лиувилля // Вестн. МГУ. Сер. 1. Матем., мех. 1990. №6. С. 75-78.

[4] Егоров Ю. В., Кондратьев В. А. Об оценке главного собственного значения оператора Штурма-Лиувилля // Вестн. МГУ. Сер. 1. Матем., мех. 1991. №6. С. 5-11.

[5] Egorov Yu. V., Kondratjev V. A. On a Lagrange problem // C. R. Acad. Sci. Paris. Sér. I. Math. 1993. V. 317. P. 903-918.

[6] Никитин Я. Ю. Асимптотическая эффективность непараметрических критериев. М.: Наука, 1995 .

[7] Алексеев В. М., Тихомиров В. М., Фомин С. В. Оптимальное управление. М.: Наука, 1979.

[8] Ллпунов А. М. Исследование одного из особенных случаев задачи об устойчивости движения // Собр. сочинений. Т. 2. М.-Л.: Изд-во АН СССР, 1956.

[9] Ниренберг Л. Лекции по нелинейному функциональному анализу. М.: Мир, 1977.

[10] Dacorogna B., Ganbo W., Subiá N. Sur une généralization de l'inégalité de Wirtinger // Ann. Inst. H. Poincaré. 1992. V. 9. №1. P. 29-50.

Московский автомобильно-дорожный институт

Московский государственный университет им. М.В. Ломоносова

Поступило

Санкт-Петербургский госудаственный университет

19.12.97

E-mail : busl@madi.msk.su 